\title{
Efficacay of Topical Steroid in Treatment of Phimosis
}

\author{
Dr. Sneha Srivastava ${ }^{1}$, Dr. Shilpa Patankar ${ }^{2}$ \\ ${ }^{1}$ Surgery Resident, Department of Surgery, Bharati Vidyapeeth Deemed University Medical College, Dhankawadi, Pune - 411043, \\ Maharashtra, India \\ ${ }^{2}$ Associate Professor, Surgery Resident, Department of Surgery, Bharati Vidyapeeth Deemed University Medical College, Dhankawadi, \\ Pune - 411043, Maharashtra, India
}

\begin{abstract}
Introduction: Most cases of phimosis presenting for surgical opinion are physiological and self-limiting. The congenital phimosis is due to adhesions that attaches to the epithelial layers of the inner prepuce and the glans, preventing the retraction of the foreskin. Methods: 60 patients, under the age group 6 months to 5 years were selected whose came with complain of inability to retract prepuce. $0.05 \%$ Betamethasone ointment, thin layer was apply twice daily for a period of 3 weeks. Result was evaluated after 3 weeks of treatment and a follow up ever ymonth for 3 months. Result was assessed on the extend of retraction-complete or incomplete. Result: After treatment for 3 months $80 \%$ patients showed complete retraction, 10\% showed partial retraction and 10\% remained inconclusive as were lost during follow up. Conclusion: Topical steroid (0.05\% Betamethasone) for treatment of phimosis is safe, simple and safe procedure that avoid surgery procedure, its risks and no drug allergy.
\end{abstract}

Keywords: Congenital Phimosis Pathological phimosis, Betamethasone, Topical Steroids, Circumcision.

\section{Introduction}

Phimosis is narrowing of the preputial ring that prevents retraction of the foreskin over the glans penis. It can be of two types i.e. congenital (physiological) and acquired (pathological).

In Congenital Phimosis, the condition results from defects arising while the child is still in fetus. Physiological phimosis regresses with age, as the foreskin widens and gradually advances over the glans penis ${ }^{1}$. In Acquired Phimosis, the patient develops a phimosis ring after his birth, usually associated with pain, skin irritation, local infections, bleeding, dysuria, hematuria, frequent episodes of urinary tract infections, and weak urinary stream.

There are two types of treatment of phimosis: surgical and non-surgical. The surgical treatment of choice is circumcision $^{1}$. Several studies have suggested a new conservative approach with topical steroids such as Betamethasone of various strength. Topical steroids treats phimosis by three mechanisms. It causes thinning of skin and improves the elasticity of the foreskin by decreasing synthesis of hyaluronic acid, which has an anti -proliferative effect on the epidermis. It also inhibits the production of the mediators of skin inflammation, prostaglandins and leukotrienes. Finally, it has a lubricating effect which allows retraction of foreskin easily.

\section{Materials and Methods}

This was a descriptive case study performed on 60 patients with phimosis in age group of 6 months to 5 years. The exclusion Criteria included patients with no episode of urinary tract infection within one month prior to the initiation of treatment, vesico ureteric reflux disease and paraphimosis.Patients were treated for a period of 3 weeks. Parents were instructed to thoroughly clean the prepucial part and apply a thin layer of ointment after mild retraction and gently massaged it, twice a day. The patient was assessed whether the prepuce is retractable with complete exposure after three weeks. Patients were subsequently asked to follow up every month for three months.

\section{Observations and Results}

Age wise distribution of patients: Most of the patients were in the age group of 6-10months in this study. Here, we observed age dependent decrease in number of phimosis patients.

Table 1: Age wise distribution of patients

\begin{tabular}{|c|c|c|}
\hline Age category (month) & Number of patients & $\%$ \\
\hline $6-10$ & 22 & 36.67 \\
\hline $11-20$ & 14 & 23.33 \\
\hline $21-30$ & 12 & 20.00 \\
\hline $31-40$ & 5 & 8.33 \\
\hline $41-50$ & 4 & 6.67 \\
\hline$\geq 51$ & 3 & 5.00 \\
\hline Total Number of patients & 60 & 100 \\
\hline
\end{tabular}

Distribution of subjects according to age and grade of prepuce retraction: There was increase in the number of children with higher degree of preputial separation with increasing age(as per classification given by Kayaba et $\mathrm{al}^{13}$ )Grade I (No retraction of prepuce at all)Grade II (External urethral meatus exposure only).Grade III(Glans exposure halfway to the sulcus of the corona). 


\section{International Journal of Science and Research (IJSR) \\ ISSN (Online): 2319-7064}

Index Copernicus Value (2013): 6.14 | Impact Factor (2014): 5.611

Table 2: Distribution of subjects according to age and type of prepuce retraction

\begin{tabular}{|c|c|c|c|c|c|c|c|c|}
\hline & \multicolumn{2}{|c|}{ Total } & \multicolumn{2}{c|}{ Type I } & \multicolumn{2}{c|}{ Type II } & \multicolumn{2}{c|}{ Type III } \\
\hline & No. of Patients & $\%$ & No. of Patients & $\%$ & No .of Patients & $\%$ & No. of Patients & $\%$ \\
\hline 0-6 months & 6.00 & 10.00 & 6.00 & 10.00 & 0.00 & 0.00 & 0.00 & 0.00 \\
\hline 7-12 months & 21.00 & 35.00 & 21.00 & 35.00 & 0.00 & 0.00 & 0.00 & 0.00 \\
\hline 1-2 years & 17.00 & 28.33 & 17.00 & 28.33 & 0.00 & 0.00 & 0.00 & 0.00 \\
\hline 2-4 years & 13.00 & 21.67 & 9.00 & 15.00 & 3.00 & 5.00 & 1.00 & 1.67 \\
\hline 4-6 years & 3.00 & 5.00 & 1.00 & 1.67 & 2.00 & 3.33 & 0.00 & 0.00 \\
\hline
\end{tabular}

Treatment correlation with grading of phimosis: We found that $91.84 \%$ patients of grade I preputial retractability did not required surgery. Half of the patients of grade II preputial retractability showed surgery treatment requirement and half of the patients did not. Grade III patient did not required surgery treatment approach.

Table 3: Grade of preputial retractability and Surgery requirement

\begin{tabular}{|c|c|c|c|c|}
\hline \multirow{2}{*}{ Preputial retractability } & \multicolumn{5}{|c|}{ Surgery treatment } \\
\cline { 2 - 5 } & No. of patients & $\%$ & $\%$ \\
\hline Grade & YES & NO & YES & NO \\
\hline Grade I & 4 & 45 & 8.16 & 91.84 \\
\hline Grade II & 2 & 2 & 50 & 50 \\
\hline Grade III & 0 & 1 & 0 & 100 \\
\hline
\end{tabular}

Treatment outcome: We analyzed retraction after three months of treatment of the 60 patients, complete retraction was obtained in 48 patients (80\%) while partial retraction was obtained in 6 patients (10\%). However, 6 patients (10\%) remained inconclusive due to loss of follow-up.

Table 4: Treatment outcome

\begin{tabular}{|c|c|c|c|}
\hline Time & $\begin{array}{c}\text { Number of } \\
\text { patient }\end{array}$ & $\begin{array}{c}\text { Surgery required in } \\
\text { Number of patient }\end{array}$ & $\%$ \\
\hline Complete retraction & 48 & 0 & 80 \\
\hline Partial retraction & 06 & 6 & 10 \\
\hline Inconclusive & 06 & NA & 10 \\
\hline
\end{tabular}

NA not applicable as lost follow-up of patients.

Drug Allergy: In our study, 54 out of total 60 patients were completed the treatment. We did not report drug allergy to any of the study patients.

Table 5: Drug allergy

\begin{tabular}{|c|c|c|}
\hline Drug Allergy & Number of patients & $\%$ \\
\hline Absent & 54 & 100 \\
\hline Present & 0 & 0 \\
\hline
\end{tabular}

\section{Discussion}

It is important to distinguish the normal non-retractability of the foreskin due to the physiological adherence of its undersurface to the glans penis from phimosis. When these congenital adhesions alone are present, the foreskin can be retracted back sufficiently to expose the urethral meatus and adjacent glans penis. In the most severe cases, absolutely no retraction can be achieved, and there may be only a tiny pinhole opening through which urine can escape. Nonetheless, a tight foreskin may manifest with varied symptoms such as itching, smegma deposits, straining, ballooning, balanoposthitis, dysuria or a urinary infection. It is in such situations that the phimosis is termed symptomatic or pathological.
Age and Phimosis :Gairdner ${ }^{2}$ reported that $50 \%$ of foreskins are not fully retractable by 1 year of age, and $20 \%$ by 2 years of age. Phimosis declines with advancing age ${ }^{2}$. Similar trend was observed in our study, wherein increase in age showed decrease in phimosis cases. Most of the patients were in the age group of 6-10months, i.e. 22 in number. 11-20months age group had 14 (23.33\%) and 21-30 months age group had $12(20 \%)$ number of patients. Rest of age group had less number of patients i.e.5(8.33\%)in age group 31-40,4 (6.66\%) in age group 41-50 months and $3(5 \%)$ in age group more than 50 years, respectively. Here, we observed age dependent decrease in number of phimosis patients.

Extend of prepucial retraction according to age group: According to Wright, ${ }^{3}$ the prepuce is designed to be nonretractable in infancy and early childhood when the developing glans needs protection from the mechanical trauma due to clothing and chemical trauma of ammonical urine and he stressed that the time to pull the foreskin back is when the child is old enough to do it himself. Gairdner ${ }^{2}$ found that of the newborns, only $4 \%$ had fully retractable prepuce while in $42 \%$, even the tip of the external meatus could not be uncovered. In our study, the prepuce could not be retracted at all so as to make even the external urethral meatus visible in $35 \%$ children aged 7-12 months while this decreased to only $1.67 \%$ in children aged $4-6$ years. There was increase in the number of children with higher degree of preputial separation with increasing age. As the age was increased, number of patients in grade I was decreased. There was increase in the number of children with higher degree of preputial separation with increasing age.

Treatment charting in correlation with grading of phimosis and age:We found that 4 patients of grade I phimosis required surgery because the treatment was ineffective on these patients. Only 2 patients of grade II phimosis underwent surgery. Grade III phimosis patients did not required surgery treatment approach. The may be because there was increase in the number of children with higher degree of preputial separation with increasing age. As the age was increased, number of patients in grade I phimosis was decreased. However this study does not show any significant co relation with grading of phimosis with age and treatment.

Topical Steroids for phimosis treatment :Given the morbidity of circumcision, a non-surgical method of dealing with phimosis might be an alternative. Topical steroids could be used as a first-line treatment for pathologic phimosis and is a viable option prior to surgery. Previous studies have shown that local application and even injection of steroids into the prepuce reduces or abolishes phimosis, and in some cases can reverse the process of balanitis xerotica obliterans ${ }^{4,5}$. However, patients with $\mathrm{BXO}$ respond poorly to topical 


\section{International Journal of Science and Research (IJSR) \\ ISSN (Online): 2319-7064}

Index Copernicus Value (2013): 6.14 | Impact Factor (2014): 5.611

steroids. This may serve as a screening tool in such cases. In our study we did not receive any Balanitis xerotica Obliterans patients; hence we were unable to conclude same.

In this study, all the patients had taken treatment for first, second and third week, however 3 patients left from the study and total 57 patients had taken the treatment for total of 3 weeks. Patients were further followed for up monthly up for 3 months. On subsequent follow-up, we lost 3 more patients and remaining 54 patients were assessed for the final outcome. Of the 60 patients, complete retraction (fully retractable prepuce) was obtained in 48 patients (80\%) while partial retraction was obtained in 6 patients (10\%). 6 patients (10\%) remain inconclusive due to loss of follow-up. From this study, we observed that only 06 patients (10\%) partially responded to topical steroid application, hence required surgery.48 (80\%) were treated and did not underwent any surgical procedure. It can be assumed that topical application of steroid is efficient for the treatment of phimosis.

Overall, studies using topical creams for phimosis have yielded dramatic results. Efficacy figures range from 65 to $95 \%{ }^{6}$. Betamethasone $0.05 \%$ applied twice a day over a 3week period has consistently shown good results ${ }^{7-10}$. Success rate was higher in patients with no infection. Studies carried out in younger children have also yielded good results ${ }^{16}$. $0.1 \%$ betamethasone cream usage also generated comparable results.

\section{Conclusion}

- Local application of $0.05 \%$ Betamethasone is safe and effective treatment for patients of phimosis.

- Tropical steroid application is cost-effective therapy for treatment of phimosis. The age of the patient, type and severity of phimosis, application of the ointment and compliance with treatment, on a regular basis contribute to outcome of the treatment.

- Topical steroid treatment avoids potential complications of penile surgery, including anaesthesia-related risks.

- Prepucial skin is preserved in this study that can be used as grafts in many procedures.

\section{References}

[1] Mohapatra T, Kumar S. Concurrent preputial calculi and penile carcinoma--a rare association. Postgraduate medical journal. 1989;65(762):256-257.

[2] Gairdner D. Fate of the Foreskin. Br Med J (Clin Res Ed). 1949;2(4642):1433-1437.

[3] Wright J. Further to" the further fate of the foreskin". Update on the natural history of the foreskin. The Medical Journal of Australia. 1994;160(3):134-135.

[4] Tan HL. Foreskin fallacies and phimosis. Annals of the Academy of Medicine, Singapore. 1985;14(4):626-630.

[5] Poynter JH, Levy J. Balanitis xerotica obliterans: effective treatment with topical and sublesional corticosteroids. Br J Urol. 1967;39(4):420-425.

[6] Steadman B, Ellsworth P. To circ or not to circ: indications, risks, and alternatives to circumcision in the pediatric population with phimosis. Urol Nurs. 2006;26(3):181-194.
[7] Monsour MA, Rabinovitch HH, Dean GE. Medical management of phimosis in children: our experience with topical steroids. J Urol. 1999;162(3 Pt 2):11621164.

[8] Orsola A, Caffaratti J, Garat JM. Conservative treatment of phimosis in children using a topical steroid. Urology. 2000;56(2):307-310.

[9] Golubovic Z, Milanovic D, Vukadinovic V, et al. The conservative treatment of phimosis in boys. Br J Urol. 1996;78(5):786-788.

[10] Elmore JM, Baker LA, Snodgrass WT. Topical Steroid Therapy as an Alternative to Circumcision for Phimosis in Boys Younger Than 3 Years. The Journal of Urology. 2002;168(4, Supplement):1746-1747. 